Bull. Korean Math. Soc. 50 (2013), No. 6, pp. 2089-2101

http://dx.doi.org/10.4134/BKMS.2013.50.6.2089

\title{
REAL HYPERSURFACES IN A NON-FLAT COMPLEX SPACE FORM WITH LIE RECURRENT STRUCTURE JACOBI OPERATOR
}

\author{
George Kaimakamis and Konstantina Panagiotidou
}

\begin{abstract}
The aim of this paper is to introduce the notion of Lie recurrent structure Jacobi operator for real hypersurfaces in non-flat complex space forms and to study such real hypersurfaces. More precisely, the non-existence of such real hypersurfaces is proved.
\end{abstract}

\section{Introduction}

A complex space form is an $n$-dimensional Kaehler manifold of constant holomorphic sectional curvature $c$ and it is denoted by $M_{n}(c)$. A complete and simply connected complex space form is complex analytically isometric to

- a complex projective space $\mathbb{C} P^{n}$ if $c>0$,

- a complex Euclidean space $\mathbb{C}^{n}$ if $c=0$,

- or a complex hyperbolic space $\mathbb{C} H^{n}$ if $c<0$.

Let $M$ be a real hypersurface in non-flat complex space form $M_{n}(c), c \neq$ 0 . Then an almost contact metric structure $(\varphi, \xi, \eta, g)$ can be defined on $M$ induced from the Kaehler metric $G$ and the complex structure $J$ on $M_{n}(c)$. The structure vector field $\xi$ is called principal if $A \xi=\alpha \xi$, where $A$ is the shape operator of $M$ and $\alpha=\eta(A \xi)$ is a smooth function. A real hypersurface is said to be a Hopf hypersurface if $\xi$ is principal.

The study of real hypersurfaces in $M_{n}(c), c \neq 0$, is a classical problem in the area of Differential Geometry. In [10], [11] Takagi was the first who studied and classified homogeneous real hypersurfaces in $\mathbb{C} P^{n}$ and showed that they could be divided into six types, namely $\left(A_{1}\right),\left(A_{2}\right),(B),(C),(D)$ and $(E)$. In the case of $\mathbb{C} H^{n}$, Berndt in [1] classified real hypersurfaces with constant principal curvatures, when $\xi$ is principal. Such real hypersurfaces are homogeneous. Recently, Berndt and Tamaru in [2] have given a complete classification of homogeneous real hypersurfaces in $\mathbb{C} H^{n}, n \geq 2$.

Received January 11, 2013; Revised March 15, 2013.

2010 Mathematics Subject Classification. Primary 53C40; Secondary 53C15, 53D15.

Key words and phrases. real hypersurface, structure Jacobi operator, Lie recurrent, nonflat complex space forms. 
The Jacobi operator with respect to $X$ on $M$ is defined by $R(\cdot, X) X$, where $R$ is the Riemmanian curvature of $M$. For $X=\xi$ the Jacobi operator is called structure Jacobi operator and is denoted by $l=R(\cdot, \xi) \xi$. It has a fundamental role in almost contact manifolds. Many researchers have studied real hypersurfaces in terms of the structure Jacobi operator.

The Lie derivative of the structure Jacobi operator is an issue, which has been extensively studied. More precisely, in [6] Pérez and Santos proved the non-existence of real hypersurfaces in $\mathbb{C} P^{n}, n \geq 3$, whose structure Jacobi operator is Lie parallel, i.e., $\mathcal{L}_{X} l=0$ for any $X \in T M$. On the other hand, real hypersurfaces in $\mathbb{C} P^{n}, n \geq 3$, equipped with Lie $\xi$-parallel structure Jacobi operator, i.e., $\mathcal{L}_{\xi} l=0$, are classified by Pérez et al. in [8]. Ivey and Ryan in [3] extend some of the above results in $\mathbb{C} P^{2}$ and $\mathbb{C} H^{2}$. More precisely, they proved that in $\mathbb{C} P^{2}$ and $\mathbb{C} H^{2}$ no real hypersurfaces whose structure Jacobi operator is Lie parallel exist, but real hypersurfaces in $\mathbb{C} P^{2}$ and $\mathbb{C} H^{2}$, whose structure Jacobi operator is Lie $\xi$-parallel exist and gave a classification of them. Additionally, they proved that no real hypersurfaces in $\mathbb{C} P^{n}$ or $\mathbb{C} H^{n}$, $n \geq 3$, equipped with Lie parallel structure Jacobi operator exist. Recently, in [9] Pérez and Suh studied the condition of Lie $\mathbb{D}$-parallel structure Jacobi operator, i.e., $\mathcal{L}_{X} l=0$, where $X$ is orthogonal to $\xi$. They proved that no Hopf real hypersurfaces in $\mathbb{C} P^{n}, n \geq 3$, satisfying the previous condition exist. Extending the previous work, in [5] the non-existence of three dimensional real hypersurfaces in non-flat complex space forms, whose structure Jacobi operator is Lie $\mathbb{D}$-parallel was proved.

Generally, a tensor field $P$ of type $(1,1)$ on $M$ is called recurrent if a 1-form $\omega$ on $M$ exists and the following relation is satisfied $\left(\nabla_{X} P\right) Y=\omega(X) P(Y), X$, $Y$ tangent to $M$. The condition of recurrent structure Jacobi operator has been studied. More precisely in [7] the non-existence of real hypersurfaces in $\mathbb{C} P^{n}$, $n \geq 3$, whose structure Jacobi operator is recurrent is proved. Furthermore, in [12] is proved that no three dimensional real hypersurfaces in non-flat complex space forms equipped with recurrent structure Jacobi operator exist.

Motivated by all the above the following question raises naturally:

Question. Are there real hypersurfaces in non-flat complex space forms with Lie recurrent structure Jacobi operator?

First of all, we call the structure Jacobi operator of a real hypersurface Lie recurrent, when the following relation is satisfied

$$
\left(\mathcal{L}_{X} l\right) Y=\omega(X) l Y,
$$

where $X, Y \in T M$ and $\omega$ is a 1 -form.

In this paper, we suppose that $\omega \neq 0$, because if $\omega=0$, then $\mathcal{L}_{X} l=0$ and this is the Lie parallelness condition. The following result is obtained and proved:

Main Theorem. There exist no real hypersurfaces in $M_{n}(c), n \geq 2$ and $c \neq 0$, whose structure Jacobi operator is Lie recurrent. 
It would be interesting to study also the condition of Lie recurrency for the shape operator $A$, i.e., $\left(\mathcal{L}_{X} A\right) Y=\omega(X) A Y$, or the structure tensor $\varphi$, i.e., $\left(\mathcal{L}_{X} \varphi\right) Y=\omega(X) \varphi Y$. Furthermore, the Lie $\mathbb{D}$-recurrency is another issue which appears appealing to be studied, i.e., $\left(\mathcal{L}_{X} P\right) Y=\omega(X) P Y$, where $X$ orthogonal to $\xi, Y \in T M$ and $P$ is a tensor field of type $(1,1)$.

\section{Preliminaries}

Throughout this paper all manifolds, vector fields etc. are assumed to be of class $C^{\infty}$ and all manifolds are assumed to be connected. Furthermore, the real hypersurfaces are supposed to be oriented and without boundary. Let $M$ be a real hypersurface immersed in a non-flat complex space form $\left(M_{n}(c), G\right)$ with complex structure $J$ of constant holomorphic sectional curvature $c$. Let $N$ be a unit normal vector field on $M$ and $\xi=-J N$. For a vector field $X$ tangent to $M$ we can write $J X=\varphi X+\eta(X) N$, where $\varphi X$ and $\eta(X) N$ are the tangential and the normal component of $J X$, respectively. The Riemannian connections $\bar{\nabla}$ in $M_{n}(c)$ and $\nabla$ in $M$ are related for any vector fields $X, Y$ on $M$ :

$$
\begin{aligned}
& \bar{\nabla}_{Y} X=\nabla_{Y} X+g(A Y, X) N, \\
& \bar{\nabla}_{X} N=-A X,
\end{aligned}
$$

where $g$ is the Riemannian metric induced from the metric $G$ and $A$ is the shape operator of $M$ in $M_{n}(c)$ with respect to $N$. M has an almost contact metric structure $(\varphi, \xi, \eta, g)$ induced from $J$ on $M_{n}(c)$ where $\varphi$ is a $(1,1)$ tensor field and $\eta$ a 1-form on $M$ such that

$$
g(\varphi X, Y)=G(J X, Y), \quad \eta(X)=g(X, \xi)=G(J X, N) .
$$

Then we have

$$
\begin{aligned}
& \varphi^{2} X=-X+\eta(X) \xi, \quad \eta \circ \varphi=0, \quad \varphi \xi=0, \quad \eta(\xi)=1 \\
& g(\varphi X, \varphi Y)=g(X, Y)-\eta(X) \eta(Y), \quad g(X, \varphi Y)=-g(\varphi X, Y), \\
& \nabla_{X} \xi=\varphi A X, \quad\left(\nabla_{X} \varphi\right) Y=\eta(Y) A X-g(A X, Y) \xi .
\end{aligned}
$$

Since the ambient space is of constant holomorphic sectional curvature $c$, the Gauss and Codazzi equations are respectively given by

$$
\begin{gathered}
R(X, Y) Z=\frac{c}{4}[g(Y, Z) X-g(X, Z) Y+g(\varphi Y, Z) \varphi X-g(\varphi X, Z) \varphi Y \\
-2 g(\varphi X, Y) \varphi Z]+g(A Y, Z) A X-g(A X, Z) A Y, \\
\left(\nabla_{X} A\right) Y-\left(\nabla_{Y} A\right) X=\frac{c}{4}[\eta(X) \varphi Y-\eta(Y) \varphi X-2 g(\varphi X, Y) \xi],
\end{gathered}
$$

where $R$ denotes the Riemannian curvature tensor on $M$ and $X, Y, Z$ are any vector fields on $M$.

Relation (2.2) implies that the structure Jacobi operator $l$ is given by:

$$
l X=\frac{c}{4}[X-\eta(X) \xi]+\alpha A X-\eta(A X) A \xi,
$$

where $\alpha=\eta(A \xi)$. 
For every point $P \in M$, the tangent space $T_{P} M$ can be decomposed as following:

$$
T_{P} M=\operatorname{span}\{\xi\} \oplus \mathbb{D},
$$

where $\mathbb{D}=\left\{X \in T_{P} M: \eta(X)=0\right\}$. Due to the above decomposition, the vector field $A \xi$ can be written:

$$
A \xi=\alpha \xi+\beta U
$$

where $\beta=\left|\varphi \nabla_{\xi} \xi\right|$ and $U=-\frac{1}{\beta} \varphi \nabla_{\xi} \xi \in \operatorname{ker}(\eta)$, provided that $\beta \neq 0$.

\section{Case of real hypesurfaces in $M_{n}(c), n \geq 3$ and $c \neq 0$}

In this section, the symbol $M_{n}(c)$ is used to denote $\mathbb{C} P^{n}$ and $\mathbb{C} H^{n}, n \geq 3$. Let $M$ be a real hypersurface in $M_{n}(c)$, whose structure Jacobi operator is Lie recurrent.

We consider the open subset $\mathcal{N}$ of $M$ such that

$$
\mathcal{N}=\{P \in M: \beta \neq 0 \text { in a neighborhood of } P\} .
$$

Furthermore, we consider $\mathcal{V}, \Omega$ open subsets of $\mathcal{N}$ such that

$$
\begin{aligned}
& \mathcal{V}=\{P \in \mathcal{N}: \alpha=0 \text { in a neighborhood of } P\}, \\
& \Omega=\{P \in \mathcal{N}: \alpha \neq 0 \text { in a neighborhood of } P\},
\end{aligned}
$$

where $\mathcal{V} \cup \Omega$ is open and dense in the closure of $\mathcal{N}$.

Relation (1.1) more analytically is written

$$
\nabla_{X}(l Y)-\nabla_{l Y} X-l \nabla_{X} Y+l \nabla_{Y} X=\omega(X) l Y .
$$

Lemma 3.1. Let $M$ be a real hypersurface in $M_{n}(c)$, whose structure Jacobi operator is Lie recurrent. Then $\mathcal{V}$ is empty.

Proof. In $\mathcal{V}$ relation (2.5) becomes $A \xi=\beta U$. From (2.4) for $X=\varphi U$ and $X=\xi$ we obtain $l \varphi U=\frac{c}{4} \varphi U$ and $l \xi=0$. Furthermore, the first of (2.1) implies $\nabla_{\xi} \xi=\beta \varphi U$.

Relation (3.1) for $X=\xi$ and $Y=\varphi U$, due to the first (2.1) yields

$$
\frac{c}{4} \nabla_{\xi} \varphi U-\frac{c}{4} \varphi A \varphi U-l \nabla_{\xi} \varphi U+l \varphi A \varphi U=\frac{c}{4} \omega(\xi) \varphi U .
$$

The inner product of the last one with $\xi$, due to $l \xi=0$ and $\nabla_{\xi} \xi=\beta \varphi U$, results in $c=0$, which is a contradiction and this completes the proof the present Lemma.

Lemma 3.2. Let $M$ be a real hypersurface in $M_{n}(c)$, whose structure Jacobi operator is Lie recurrent. Then $\Omega$ is empty.

Proof. The inner product of relation (3.1) with $\xi$, since $l \xi=0$ and the first of (2.1) implies

$$
g(l \varphi A X+l A \varphi X, Y)+l Y[g(X, \xi)]=0, X, Y \in T M .
$$


Relation (3.2) for $X=\xi$, due to (2.5), yields: $g(l \varphi U, Y)=0$ for any $Y \in$ $T M$ and this results in $l \varphi U=0$. Then relation (2.4) for $X=\varphi U$ implies:

$$
A \varphi U=-\frac{c}{4 \alpha} \varphi U
$$

Owing to (3.3) we have that $g(A U, \varphi U)=g(A \varphi U, U)=0$ and $g(A \varphi U, Z)=$ $g(A Z, \varphi U)=0$ for any $Z \in \mathbb{D}_{U}$, where $\mathbb{D}_{U}$ is the orthogonal complement to $\operatorname{span}\{\xi, U, \varphi U\}$.

Suppose that $A U=\gamma U+\beta \xi+\kappa Z$, where $\gamma=g(A U, U), \kappa=g(A U, Z)=$ $g(A Z, U)$ and $Z \in \mathbb{D}_{U}$. Relation (2.4) for $X=U$ and $X=Z$, because of the latter yields

$$
l U=\left(\frac{c}{4}+\alpha \gamma-\beta^{2}\right) U+\alpha \kappa Z \text { and } l Z=\frac{c}{4} Z+\alpha A Z .
$$

Relation (3.2) for $X=U$, due to $l \varphi U=0, A U=\gamma U+\beta \xi+\kappa Z$ and (3.3) implies: $g(\kappa l \varphi Z, Y)=0$ for any $Y \in T M$ and this leads to $\kappa l \varphi Z=0$.

Let $\Omega_{1}$ be the open subset of $\Omega$ such that

$$
\Omega_{1}=\{P \in \Omega: \kappa \neq 0 \text { in a neighborhood of } P\} .
$$

Then in $\Omega_{1}$ we have $l \varphi Z=0$ and relation (2.4) for $X=\varphi Z$ yields: $A \varphi Z=$ $-\frac{c}{4 \alpha} \varphi Z$.

Relation (3.2) for $X=\varphi U$ and $X=\varphi Z$, due to (3.3) and $A \varphi Z=-\frac{c}{4 \alpha} \varphi Z$, implies: $g\left(\frac{c}{4 \alpha} l U-l A U, Y\right)=0$ and $g\left(\frac{c}{4 \alpha} l Z-l A Z, Y\right)=0$ for any $Y \stackrel{4 \alpha}{\in} T M$ respectively. From the last two relations we obtain

$$
l A U=\frac{c}{4 \alpha} l U \text { and } l A Z=\frac{c}{4 \alpha} l Z .
$$

The inner product of the first of the above relations with $Z$, because of (3.4) and $A U=\gamma U+\beta \xi+\kappa Z$ implies: $g(A Z, Z)=-\gamma$ and the inner product of the second with $U$ taking into account the latter and (3.4) yields $\beta=0$, which is a contradiction. Therefore, $\Omega_{1}$ is empty.

So in $\Omega$ we have $\kappa=0$ and the following holds

$$
A U=\gamma U+\beta \xi, A \varphi U=-\frac{c}{4 \alpha} \varphi U, l U=\left(\frac{c}{4}+\alpha \gamma-\beta^{2}\right) U \text { and } l \varphi U=0 .
$$

Relation (3.2) for $X=\varphi U$, due to (3.5) implies $g\left[\left(\frac{c}{4 \alpha}-\gamma\right) l U, Y\right]=0$ for any $Y \in T M$ and this results in

$$
\left(\frac{c}{4 \alpha}-\gamma\right) l U=0
$$

Let $\Omega_{2}$ be the open subset of $\Omega$ such that,

$$
\Omega_{2}=\{P \in \Omega: l U \neq 0 \text { in a neighborhood of } P\} .
$$

Then in $\Omega_{2}$ we have $\gamma=\frac{c}{4 \alpha}$ and relation (3.5) becomes

$$
A U=\frac{c}{4 \alpha} U+\beta \xi, A \varphi U=-\frac{c}{4 \alpha} \varphi U, l U=\left(\frac{c}{2}-\beta^{2}\right) U, \text { and } l \varphi U=0 .
$$


The inner product of the Codazzi equation (2.3) due to the first two relations of (3.6) implies

$$
\beta \kappa_{1}+\frac{c}{2}+\frac{c^{2}}{16 \alpha^{2}}=\frac{c \kappa_{3}}{2 \alpha}+\beta^{2} \text { for } X=U \text { and } Y=\xi \text { with } \varphi U
$$

$$
(\varphi U) \beta=\beta \kappa_{1}+\frac{c}{2}+\frac{c^{2}}{8 \alpha^{2}} \text { for } X=\varphi U \text { and } Y=\xi \text { with } U \text { due to the above, }
$$

$$
(\varphi U) \alpha=\beta\left(\alpha+\kappa_{3}+\frac{3 c}{4 \alpha}\right) \text { for } X=\varphi U \text { and } Y=\xi \text { with } \xi,
$$

where $\kappa_{1}=g\left(\nabla_{U} U, \varphi U\right)$ and $\kappa_{3}=g\left(\nabla_{\xi} U, \varphi U\right)$.

The inner product of relation (3.1) for $X=\xi$ and $Y=U$ with $\varphi U$, because of $(3.6)$ and since $g\left(\nabla_{\xi}(\varphi U), U\right)=-\kappa_{3}$ yields:

$$
\left(\frac{c}{2}-\beta^{2}\right)\left(\kappa_{3}-\frac{c}{4 \alpha}\right)=0 .
$$

Let $\Omega_{21}$ be the open subset of $\Omega_{2}$ such that

$$
\Omega_{21}=\left\{P \in \Omega_{2}: \beta^{2} \neq \frac{c}{2} \text { in a neighborhood of } P\right\} .
$$

Then in $\Omega_{21}$ we have that $\kappa_{3}=\frac{c}{4 \alpha}$ and relation (3.9) becomes

$$
(\varphi U) \alpha=\beta\left(\alpha+\frac{c}{\alpha}\right)
$$

The inner product of Codazzi equation (2.3) for $X=U$ and $Y=\varphi U$ with $U$, taking into account $(3.6),(3.9)$ and the last one yields $\kappa_{1}=-\frac{c \beta}{2 \alpha^{2}}$. From (3.1) for $X=U$ and $Y=\varphi U$ due to (3.6), we obtain

$$
l \nabla_{U} \varphi=l \nabla_{\varphi U} U .
$$

The inner product of the above relation with $U$, because of (3.6) and $\kappa_{1}=$ $g\left(\nabla_{U} U, \varphi U\right)$ leads to $\kappa_{1}=0$ and due to the above relation for $\kappa_{1}$ we obtain $c=0$, which is impossible. Therefore, $\Omega_{21}$ is empty.

So in $\Omega_{2}$ we have that $\beta^{2}=\frac{c}{2}$ and relation (3.7) becomes

$$
\beta \kappa_{1}=\frac{c \kappa_{3}}{2 \alpha}-\frac{c^{2}}{16 \alpha^{2}} .
$$

Differentiation of $\beta^{2}=\frac{c}{2}$ with respect to $\varphi U$ implies: $(\varphi U) \beta=0$. The latter, taking into account (3.8) yields: $\beta \kappa_{1}=-\frac{c}{2}-\frac{c^{2}}{8 \alpha^{2}}$. Substitution of the last one in (3.10) implies: $\kappa_{3}=-\frac{c}{8 \alpha}-\alpha$. The inner product of Codazzi equation (2.3) for $X=U$ and $Y=\varphi U$ with $U$, taking into account (3.6), (3.9) and the last one yields: $\kappa_{1}=-\frac{5 c \beta}{16 \alpha^{2}}+\frac{\beta}{2}$. Substituting the last one in (3.10) and $\kappa_{3}=-\frac{c}{8 \alpha}-\alpha$ and $\beta^{2}=\frac{c}{2}$ leads to: $\beta^{2}=12 \alpha^{2}$. Differentiating the last one with respect to $\varphi U$ and taking into account $(3.9),(\varphi U) \beta=0$ and the relation for $\kappa_{3}$, we obtain $c=0$ which is a contradiction. So $\Omega_{2}=\emptyset$. 
So in $\Omega$ we have $l U=0$ and (3.5) becomes

$$
A U=\left(\frac{\beta^{2}}{\alpha}-\frac{c}{4 \alpha}\right) U+\beta \xi, A \varphi U=-\frac{c}{4 \alpha} \varphi U, \text { and } l U=l \varphi U=0 .
$$

Let $\nabla_{\xi} U=\kappa_{3} \varphi U+\lambda_{1} Z_{1}$, where $Z_{1} \in \mathbb{D}_{U}$. Then from (3.1) for $X=U$ and $Y=\xi$, due to the latter and (2.4) for $X=Z_{1}$ we obtain $\lambda_{1}\left(\frac{c}{4} Z_{1}+\alpha A Z_{1}\right)=0$. Let $\Omega_{3}$ be the open subset of $\Omega$ such that

$$
\Omega_{3}=\left\{P \in \Omega: \lambda_{1} \neq 0 \text { in a neighborhood of } P\right\} .
$$

So in $\Omega_{3}$, we have that $A Z_{1}=-\frac{c}{4 \alpha} Z_{1}$. The inner product of the Codazzi equation taking into account $\nabla_{\xi} U=\kappa_{3} \varphi U+\lambda_{1} Z_{1}$ and (3.11) implies

$$
\begin{aligned}
& Z_{1} \alpha=\beta \lambda_{1} \text { for } X=Z_{1} \text { and } Y=\xi \text { with } \xi \\
& g\left(\nabla_{U} U, Z_{1}\right)=\frac{\beta \lambda_{1}}{\alpha} \text { for } X=U \text { and } Y=\xi \text { with } Z_{1}, \\
& Z_{1} \beta=\frac{\beta^{2} \lambda_{1}}{\alpha} \text { for } X=Z_{1} \text { and } Y=U \text { with } \xi \text { due to the previous one. }
\end{aligned}
$$

Furthermore, the inner product of the Codazzi equation for $X=Z_{1}$ and $Y=U$ with $U$ owing to (3.11) and all the above relations results in $c=0$, which is a contradiction. Therefore, $\Omega_{3}$ is empty.

So in $\Omega \lambda_{1}=0$ and $\nabla_{\xi} U=\kappa_{3} \varphi U$. The inner product of Codazzi equation, because of (3.11) yields:

$\frac{\beta^{2} \kappa_{3}}{\alpha}=\beta \kappa_{1}+\frac{c}{4 \alpha}\left(\frac{\beta^{2}}{\alpha}-\frac{c}{4 \alpha}\right)$ for $X=U$ and $Y=\xi$ with $\varphi U$,

$(\varphi U) \beta=\beta^{2}+\beta \kappa_{1}+\frac{c}{2 \alpha}\left(\frac{\beta^{2}}{\alpha}-\frac{c}{4 \alpha}\right)$ for $X=\varphi U$ and $Y=\xi$ with $U$ due to (3.12),

$(\varphi U) \alpha=\beta\left(\alpha+\kappa_{3}+\frac{3 c}{4 \alpha}\right)$ for $X=\varphi U$ and $Y=\xi$ with $\xi$,

$\xi \alpha=\frac{4 \alpha^{2} \beta \kappa_{2}}{c}$ for $X=\varphi U$ and $Y=\xi$ with $\varphi U$,

$(\varphi U)\left(\frac{\beta^{2}}{\alpha}-\frac{c}{4 \alpha}\right)=\beta\left(\frac{\beta^{2}}{\alpha}+\frac{\beta \kappa_{1}}{\alpha}-\frac{3 c}{4 \alpha}\right)$ for $X=U$ and $Y=\varphi U$ with $U$,

$U \alpha=\frac{4 \alpha \beta^{2} \kappa_{2}}{c}$ for $X=U$ and $Y=\varphi U$ with $\varphi U$,

$U \alpha=\xi \beta=\frac{4 \alpha \beta^{2} \kappa_{2}}{c}$ for $X=U$ and $Y=\xi$ with $\xi$ due to (3.17), 
$U \beta=\beta \kappa_{2}\left(\frac{4 \beta^{2}}{c}+1\right)$ for $X=U$ and $Y=\xi$ with $U$ due to (3.15) and (3.18),

where $\kappa_{1}=g\left(\nabla_{U} U, \varphi U\right), \kappa_{2}=g\left(\nabla_{\varphi U} U, \varphi U\right)$ and $\kappa_{3}=g\left(\nabla_{\xi} U, \varphi U\right)$.

Relation (3.16), because of (3.12), (3.14) and (3.13), yields:

$$
\kappa_{3}=-4 \alpha,
$$

and so relation (3.12) becomes:

$$
\beta \kappa_{1}=\frac{c}{4 \alpha}\left(\frac{c}{4 \alpha}-\frac{\beta^{2}}{\alpha}\right)-4 \beta^{2} .
$$

The Riemannian curvature on $M$ satisfies relation (2.2) and on the other hand is given by the relation $R(X, Y) Z=\nabla_{X} \nabla_{Y} Z-\nabla_{Y} \nabla_{X} Z-\nabla_{[X, Y]} Z$. The combination and the inner product of these two relations for $X=Z=U$, $Y=\xi$ with $\varphi U$ and $X=\xi, Y=\varphi U, Z=U$ and with $\varphi U$, owing to $\nabla_{\xi}(\varphi U)=$ $\left(\nabla_{\xi} \varphi\right) U+\varphi \nabla_{\xi} U$ and the second of (2.1) implies respectively:

$$
\begin{aligned}
U \kappa_{3}-\xi \kappa_{1} & =\kappa_{2}\left(\frac{\beta^{2}}{\alpha}-\frac{c}{4 \alpha}-\kappa_{3}\right), \\
(\varphi U) \kappa_{3}-\xi \kappa_{2} & =\kappa_{1}\left(\kappa_{3}+\frac{c}{4 \alpha}\right)+\beta\left(\kappa_{3}-\frac{c}{2 \alpha}\right) .
\end{aligned}
$$

Differentiating the relations (3.20) and (3.21) with respect to $U$ and $\xi$, respectively and substituting in (3.22) and due to (3.18), (3.15) and (3.20) we obtain:

$$
\kappa_{2}\left(c-2 \beta^{2}-4 \alpha^{2}\right)=0 .
$$

Owing to (3.24), let $\Omega_{4}$ be the open subset of $\Omega$ such that

$$
\Omega_{4}=\left\{P \in \Omega: \kappa_{2} \neq 0 \text { in a neighborhood of } P\right\} .
$$

So in $\Omega_{4}$ we obtain: $2 \beta^{2}+4 \alpha^{2}=c$. Differentiation of the last relation along $\xi$ and taking into account (3.18), (3.15) and $2 \beta^{2}+4 \alpha^{2}=c$ yields: $\kappa_{2}=0$, which is a contradiction. Therefore, $\Omega_{4}$ is empty.

Thus, $\kappa_{2}=0$ in $\Omega$ and relations (3.19), (3.18) and (3.15) become:

$$
U \alpha=U \beta=\xi \alpha=\xi \beta=0 .
$$

Using the above relations and (3.20) we obtain:

$$
\begin{aligned}
& {[U, \xi] \alpha=U(\xi \alpha)-\xi(U \alpha)=0} \\
& {[U, \xi] \alpha=\left(\nabla_{U} \xi-\nabla_{\xi} U\right) \alpha=\frac{1}{4 \alpha}\left(4 \beta^{2}+16 \alpha^{2}-c\right)(\varphi U) \alpha .}
\end{aligned}
$$

Combining the last two relations we have:

$$
\left(4 \beta^{2}+16 \alpha^{2}-c\right)(\varphi U) \alpha=0 .
$$

Let $\Omega_{5}$ be the open subset of $\Omega$ such that

$$
\Omega_{5}=\{P \in \Omega:(\varphi U) \alpha \neq 0 \text { in a neighborhood of } P\} .
$$


So in $\Omega_{5}$ from (3.25) we have: $16 \alpha^{2}+4 \beta^{2}=c$. Differentiating the last relation with respect to $\varphi U$ and taking into account (3.14), (3.13), (3.20), (3.21) and $c=16 \alpha^{2}+4 \beta^{2}$, implies: $\alpha^{2}=0$, which is impossible. So $\Omega_{5}$ is empty.

Hence, on $\Omega$ we have $(\varphi U) \alpha=0$. Then, relations (3.14), (3.20) and (3.21) imply: $c=4 \alpha^{2}$ and $\beta \kappa_{1}=\alpha^{2}-5 \beta^{2}$. On the other hand from relation (3.23), because of (3.20) we obtain: $\kappa_{1}=-2 \beta$. Substitution of $\kappa_{1}$ in $\beta \kappa_{1}=\alpha^{2}-$ $5 \beta^{2}$ yields: $3 \beta^{2}=\alpha^{2}$. Taking the covariant derivative along $\varphi U$ of $3 \beta^{2}=$ $\alpha^{2}$, because of (3.13), we conclude: $\beta=0$ which is a contradiction and this completes the proof of the present Lemma.

From Lemmas 3.1 and 3.2, we lead to the following result:

Proposition 3.3. Every real hypersurface in $M_{n}(c), n \geq 3$, whose structure Jacobi operator is Lie recurrent, is a Hopf hypersurface.

Since $M$ is a Hopf hypersurface, we know that $\alpha$ is constant. Let $W \in \mathbb{D}$, such that $A W=\lambda W$, then $\left(\lambda-\frac{\alpha}{2}\right) A \varphi W=\left(\frac{\lambda \alpha}{2}+\frac{c}{4}\right) \varphi W$ at some point $P \in$ M.

- Case I: $\alpha^{2}+c \neq 0$.

In this case we have that $\lambda \neq \frac{\alpha}{2}$ so $A \varphi W=\nu \varphi W$, where $\nu=\frac{2 \lambda \alpha+c}{4 \lambda-2 \alpha}$. The following relation holds on $M$ (Corollary $2.3[4]$ ):

$$
\lambda \nu=\frac{\alpha}{2}(\lambda+\nu)+\frac{c}{4} .
$$

The first of relation (2.1) and relation (2.4) implies respectively

$$
\begin{aligned}
& \nabla_{W} \xi=\lambda \varphi W \quad \text { and } \quad \nabla_{\varphi W} \xi=-\nu W, \\
& l W=\left(\frac{c}{4}+\alpha \lambda\right) W \text { and } l \varphi W=\left(\frac{c}{4}+\alpha \nu\right) \varphi W .
\end{aligned}
$$

The inner product of relation (3.1) for $X=W$ and $Y=\varphi W$ with $\xi$, taking into account (3.27) and (3.28) implies

$$
(\lambda+\nu)\left(\frac{c}{4}+\alpha \nu\right)=0 .
$$

Due to the above relation we consider $M_{1}$ be the open subset of $M$ such that:

$$
M_{1}=\{P \in M: \lambda \neq-\nu \text { in a neighborhood of } P\} .
$$

Then on $M_{1}$ we have that $\alpha \nu=-\frac{c}{4}$. The inner product of relation (3.1) with $\xi$ for $X=\varphi W$ and $Y=W$ due to (3.27) and (3.28) yields: $\alpha \lambda=-\frac{c}{4}$. Substitution of the last two relations in (3.26) leads to $\lambda \nu=0$. Suppose that $\nu \neq 0$ then $\lambda=0$ and relation $\alpha \lambda=-\frac{c}{4}$ results in $c=0$, which is a contradiction. So $\nu=0$ and following the same procedure as in the previous case we lead again to a contradiction. So $M_{1}=\emptyset$.

Therefore on $M$ relation $\lambda=-\nu$ holds.

Substitution of $\lambda=-\nu$ in (3.26) implies $c=-4 \lambda^{2}$. So we conclude that $c<0$ and that $\lambda, \nu$ are constant. The Hopf real hypersurface which satisfies 
the previous conditions is that of type $B$ in $\mathbb{C} H^{n}$. Substituting the eigenvalues of it in $\lambda=-\nu$ leads to a contradiction (for the eigenvalues see [1]).

- Case II: $\alpha^{2}+c=0$.

In this case we have that $\alpha \neq 0$, because if $\alpha=0$, then $c=0$, which is impossible. First we suppose that $\lambda \neq \frac{\alpha}{2}$ and from relation (3.26), owing to $\alpha^{2}+c=0$, we obtain that $\nu=\frac{\alpha}{2}$, where $\nu$ is defined as in the previous case. The inner product of relation (3.1) for $X=W$ and $Y=\varphi W$ with $\xi$, taking into account (3.27) and (3.28) implies: $\lambda=-\frac{\alpha}{2}$. The inner product of relation (3.1) for $X=W$ and $Y=\xi$ with $\varphi W$, because of (3.27) and (3.28), yields $g\left(\nabla_{\xi} W, \varphi W\right)=-\frac{\alpha}{2}$, and for $X=\varphi W$ and $Y=\xi$ with $W$, because of (3.27) and (3.28), implies $g\left(\nabla_{\xi} W, \varphi W\right)=\frac{\alpha}{2}$. The combination of the last two relations results in $\alpha=0$, which is impossible.

So we examine the remaining case of $\lambda=\frac{\alpha}{2}$. That will be the only eigenvalue for all vectors in $\mathbb{D}$. The inner product of relation (3.1) for $X=W$ and $Y=\varphi W$ with $\xi$, taking into account (3.27) and (3.28) and that the only eigenvalue is $\frac{\alpha}{2}$ implies $\alpha=0$, which is impossible.

Therefore we have proved that there exist no real hypersurfaces in complex space forms of dimension higher than or equal to 3 , whose structure Jacobi operator is Lie recurrent.

\section{Case of real hypersurfaces in $M_{n}(c), n=2$ and $c \neq 0$}

Let $M$ be a non-Hopf hypersurface in $M_{2}(c), c \neq 0$. We consider a local orthonormal basis $\{U, \varphi U, \xi\}$. Then the following lemma holds.

Lemma 4.1 ([5]). Let $M$ be a real hypersurface in $M_{2}(c)$. Then the following relations hold on $M$

$$
\begin{aligned}
& A U=\gamma U+\delta \varphi U+\beta \xi, \quad A \varphi U=\delta U+\mu \varphi U, \\
& \nabla_{U} \xi=-\delta U+\gamma \varphi U, \quad \nabla_{\varphi U} \xi=-\mu U+\delta \varphi U, \quad \nabla_{\xi} \xi=\beta \varphi U, \\
& \nabla_{U} U=\kappa_{1} \varphi U+\delta \xi, \quad \nabla_{\varphi U} U=\kappa_{2} \varphi U+\mu \xi, \quad \nabla_{\xi} U=\kappa_{3} \varphi U, \\
& \nabla_{U} \varphi U=-\kappa_{1} U-\gamma \xi, \quad \nabla_{\varphi U} \varphi U=-\kappa_{2} U-\delta \xi, \quad \nabla_{\xi} \varphi U=-\kappa_{3} U-\beta \xi
\end{aligned}
$$

where $\gamma, \delta, \mu, \kappa_{1}, \kappa_{2}, \kappa_{3}$ are smooth functions on $M$.

We suppose that structure Jacobi operator is Lie recurrent. We consider the open subset $\mathcal{N}$ of $M$ such that

$$
\mathcal{N}=\{P \in M: \beta \neq 0 \text { in a neighborhood of } P\} .
$$

Furthermore, we consider $\mathcal{V}, \Omega$ open subsets of $\mathcal{N}$ such that

$$
\begin{aligned}
& \mathcal{V}=\{P \in \mathcal{N}: \alpha=0 \text { in a neighborhood of } P\}, \\
& \Omega=\{P \in \mathcal{N}: \alpha \neq 0 \text { in a neighborhood of } P\},
\end{aligned}
$$

where $\mathcal{V} \cup \Omega$ is open and dense in the closure of $\mathcal{N}$.

In this case also relation (3.1) holds. 
Lemma 4.2. Let $M$ be a real hypersurface in $M_{2}(c)$, whose structure Jacobi operator is Lie recurrent. Then $\mathcal{V}$ is empty.

Proof. In $\mathcal{V}$ relation (2.5) becomes $A \xi=\beta U$. From (2.4) for $X=\varphi U$ and $X=\xi$ we obtain $l \varphi U=\frac{c}{4} \varphi U$ and $l \xi=0$. Furthermore, the first of (2.1) implies $\nabla_{\xi} \xi=\beta \varphi U$.

Relation (3.1) for $X=\xi$ and $Y=\varphi U$, due to the first (2.1) yields:

$$
\frac{c}{4} \nabla_{\xi} \varphi U-\frac{c}{4} \varphi A \varphi U-l \nabla_{\xi} \varphi U+l \varphi A \varphi U=\frac{c}{4} \omega(\xi) \varphi U .
$$

The inner product of the last one with $\xi$, due to $l \xi=0$ and $\nabla_{\xi} \xi=\beta \varphi U$, results in $c=0$, which is a contradiction and this completes the proof the present Lemma.

Next we work on $\Omega$.

Lemma 4.3. Let $M$ be a real hypersurface in $M_{2}(c)$, whose structure Jacobi operator is Lie recurrent. Then $\Omega$ is empty.

Proof. The inner product of relation (3.1) with $\xi$ implies

$$
g(l \varphi A X+l A \varphi X, Y)+l Y[g(X, \xi)]=0, X, Y \in T M .
$$

Relation (4.2) for $X=\xi$, due to (2.5), yields: $g(l \varphi U, Y)=0$ for any $Y \in$ $T M$ and this results in $l \varphi U=0$. Then relation (2.4) for $X=\varphi U$ implies:

$$
A \varphi U=-\frac{c}{4 \alpha} \varphi U
$$

So relation (4.1) becomes

$$
A U=\gamma U+\beta \xi, \quad A \varphi U=-\frac{c}{4 \alpha} \varphi U .
$$

Relation (4.2) for $X=\varphi U$, due to (4.3) implies: $g\left[\left(\frac{c}{4 \alpha}-\gamma\right) l U, Y\right]=0$ for any $Y \in T M$ and this results in

$$
\left(\frac{c}{4 \alpha}-\gamma\right) l U=0 .
$$

Let $\Omega_{1}$ be the open subset of $\Omega$ such that,

$$
\Omega_{1}=\{P \in \Omega: l U \neq 0 \text { in a neighborhood of } P\} .
$$

Then in $\Omega_{1}$ we have $\alpha \gamma=\frac{c}{4}$. Following the same procedure as in Lemma 3.2 we lead to $\Omega_{1}=\emptyset$.

So in $\Omega$ we have that $l U=0$. The last relation leads to the conclusion that the structure Jacobi operator $l$ vanishes on $\Omega$. Then from Proposition 7 in [3] we get that $\Omega$ is empty and this completes the proof of the present Lemma.

From Lemmas 4.2 and 4.3, we lead to the following result.

Proposition 4.4. Every real hypersurface in $M_{2}(c)$, whose structure Jacobi operator is Lie recurrent, is a Hopf hypersurface. 
Since $M$ is a Hopf hypersurface, due to Theorem 2.1 ([4]) we have that $\alpha$ is a constant. We consider a unit vector field $e \in \mathbb{D}$, such that $A e=\mu_{1} e$, then $A \varphi e=\nu_{1} \varphi e$ at some point $P \in M$, where $\{e, \varphi e, \xi\}$ is a local orthonormal basis. Then the following relation holds on $M$ (Corollary 2.3 [4]):

$$
\mu_{1} \nu_{1}=\frac{\alpha}{2}\left(\mu_{1}+\nu_{1}\right)+\frac{c}{4}
$$

Relation (2.4) and the first of (2.1) implies respectively:

$$
\begin{aligned}
& \nabla_{e} \xi=\mu_{1} \varphi e \text { and } \nabla_{\varphi e} \xi=-\nu_{1} e \\
& l e=\left(\frac{c}{4}+\alpha \mu_{1}\right) e \text { and } l \varphi e=\left(\frac{c}{4}+\alpha \nu_{1}\right) \varphi e .
\end{aligned}
$$

The inner product of relation (3.1) for $X=e$ and $Y=\varphi e$ with $\xi$ and for $X=\varphi e$ and $Y=e$ with $\xi$, taking into account (4.5) and (4.6) yields respectively:

$$
\begin{aligned}
& \left(\mu_{1}+\nu_{1}\right)\left(\frac{c}{4}+\alpha \nu_{1}\right)=0, \\
& \left(\mu_{1}+\nu_{1}\right)\left(\frac{c}{4}+\alpha \mu_{1}\right)=0 .
\end{aligned}
$$

Suppose that $\mu_{1}, \nu_{1}$ are distinct at point $P$. Because of (4.7) we consider $M_{2}$ the open subset of $M$ such that

$$
M_{2}=\left\{P \in M: \mu_{1} \neq-\nu_{1} \text { in a neighborhood of } P\right\} .
$$

So from (4.7) and (4.8) we obtain that $\alpha \mu_{1}+\frac{c}{4}=0$ and $\alpha \nu_{1}+\frac{c}{4}=0$. The combination of the last two relations implies $\alpha\left(\mu_{1}-\nu_{1}\right)=0$. Since $\mu_{1}, \nu_{1}$ are distinct the latter implies that $\alpha=0$ and substituting that in $\alpha \mu_{1}+\frac{c}{4}=0$ implies $c=0$, which is a contradiction. So $M_{2}=\emptyset$.

Therefore in $M$ we have that $\mu_{1}=-\nu_{1}$. Substitution in (4.4) results in $c=-4 \mu_{1}^{2}$. From the last relation we conclude that $c<0$ and $\mu_{1}=$ constant. The only hypersurface that we have in this case is of type B in $\mathbb{C} H^{2}$. Substituting the eigenvalues of this hypersurface in relation $\mu_{1}=-\nu_{1}$ leads to a contradiction (see for the eigenvalues [1]).

So the remaining case is that of $\mu_{1}=\nu_{1}$ at all points. Then from (4.7), we obtain that either $\mu_{1}=0$ or $\frac{c}{4}+\alpha \mu_{1}=0$.

- If $\mu_{1}=0$, then substitution in (4.4) implies $c=0$, which is a contradiction.

- If $\frac{c}{4}+\alpha \mu_{1}=0$, then substitution in (4.4) yields $\mu_{1}=0$. Substituting the last one in $\frac{c}{4}+\alpha \mu_{1}=0$ leads to $c=0$, which is impossible.

Therefore, no three dimensional real hypersurfaces in $M_{2}(c), c \neq 0$, exist and this completes the proof of Main Theorem.

Acknowledgments. The authors would like to express their gratitude to the referee for the careful reading of the manuscript and for the comments on improving the paper. 


\section{References}

[1] J. Berndt, Real hypersurfaces with constant principal curvatures in complex hyperbolic space, J. Reine Angew. Math. 395 (1989), 132-141.

[2] J. Berndt and H. Tamaru, Cohomogeneity one actions on noncompact symmetric spaces of rank one, Trans. Amer. Math. Soc. 359 (2007), no. 7, 3425-3438.

[3] T. A. Ivey and P. J. Ryan, The structure Jacobi operator for real hypersurfaces in $\mathbb{C} P^{2}$ and $\mathbb{C H}^{2}$, Results Math. 56 (2009), no. 1-4, 473-488.

[4] R. Niebergall and P. J. Ryan, Real hypersurfaces in complex space forms, Tight and taut submanifolds (Berkeley, CA, 1994), 233-305, Math. Sci. Res. Inst. Publ., 32, Cambridge Univ. Press, Cambridge, 1997.

[5] K. Panagiotidou Ph. J. Xenos, Real hypersurfaces in $\mathbb{C} P^{2}$ and $\mathbb{C} H^{2}$ whose structure Jacobi operator is Lie $\mathbb{D}$-parallel, Note Mat. 32 (2012) no. 2, 89-99

[6] J. D. Pérez and F. G. Santos, On the Lie derivative of structure Jacobi operator of real hypersurfaces in complex projective space, Publ. Math. Debrecen 66 (2005), no. 3-4, 269-282.

[7] _ Real hypersurfaces in complex projective space with recurrent structure Jacobi operator, Diff. Geom. And its Appl. 26 (2008), no. 2, 218-223.

[8] J. D. Pérez, F. G. Santos, and Y. J. Suh, Real hypersurfaces in complex projective space whose structure Jacobi operator is Lie $\xi$-parallel, Diff. Geom. And its Appl. 22 (2005), no. 2, 181-188.

[9] J. D. Pérez and Y. J. Suh, Real hypersurfaces in a complex projective space whose structure Jacobi operator is Lie $\mathbb{D}$-parallel, Canad. Math. Bull. 56 (2013), 306-316.

[10] R. Takagi, On homogeneous real hypersurfaces in a complex projective space, Osaka J. Math. 10 (1973), 495-506.

[11] Real hypersurfaces in a complex projective space with constant principal curvatures, J. Math. Soc. Japan 27 (1975), 43-53.

[12] Th. Theofanidis and Ph. J. Xenos, Non-existence of real hypersurfaces equipped with recurrent structure Jacobi operator in nonflat complex space forms, Results Math. 61 (2012), no. 1-2, 43-55.

George Kaimakamis

Faculty of Mathematics and Engineering Sciences

Hellenic Military ACADEmy

VAri, Attiki, Greece

E-mail address: gmiamis@gmail.com

Konstantina Panagiotidou

Mathematics Division-School of Technology

Aristotle University of Thessaloniki

ThessAloniki 54124, Greece

E-mail address: kapanagi@gen.auth.gr 